\title{
Identification of different gene expressions between diffuse- and intestinal-type spheroid-forming gastric cancer cells
}

\author{
Jong Won Lee ${ }^{1,3} \cdot$ Jae Sook Sung ${ }^{2} \cdot$ Young Soo Park ${ }^{2} \cdot$ Seok Chung ${ }^{4} \cdot$ Yeul Hong Kim ${ }^{1,2,3}$
}

Received: 14 September 2018 / Accepted: 1 February 2019 / Published online: 6 February 2019

(c) The International Gastric Cancer Association and The Japanese Gastric Cancer Association 2019

\begin{abstract}
Background Three-dimensional in vitro spheroid models are unique because they are considered for enrichment of specific cell populations with self-renewal ability. In this study, we explored the different mechanisms of gastric cancer spheroidforming cells according to the Lauren classification.

Methods We isolated and enriched cells with self-renewal ability using spheroid-forming methods from gastric cancer cell lines. The expression of candidate target genes was investigated using western blot and qRT-PCR analysis. Lentiviral shRNA knockdown of target gene expression was performed and the effects on spheroid, colony forming, and tumorigenic ability were analyzed.

Results The SNU-638, SNU-484, MKN-28, and NCI-N87 successfully formed spheroid from single cell and enriched for self-renewal ability from 11 gastric cancer cell lines, including diffuse and intestinal types. The expression of SOX2 and E-cadherin increased in spheroid-forming cells in a diffuse-type cell line (SNU-638 and SNU-484), but not in the intestinal type (MKN-28 and NCI-N87). In contrast, ERBB3 expression was only increased in intestinal-type spheroid cells. The depletion of each candidate target gene expression suppressed self-renewal ability to grow as spheroids and colonies in a soft agar assay. In particular, down-regulated ERBB3 in the intestinal-type cell lines inhibited tumor growth in a mouse xenograft model. We found that high ERBB3 gene expression correlates with decreased survival in the intestinal type of gastric cancer. Conclusions Our results suggest that diffuse- and intestinal-type spheroid-forming cells express genes differently. Our data suggest that these candidate genes from spheroid-forming cells can be used in applications in targeted therapy.
\end{abstract}

Keywords Gastric cancer · Cellular spheroid · Lauren classification · Diffuse type · Intestinal type

Yeul Hong Kim

yhk0215@korea.ac.kr

1 Brain Korea 21 Plus Project for Biomedical Science, Korea University College of Medicine, Seoul 02841, Republic of Korea

2 Cancer Research Institute, Korea University College of Medicine, Seoul 02841, Republic of Korea

3 Division of Oncology/Hematology, Department of Medicine, Korea University Anam Hospital, Korea University College of Medicine, 73 Inchon-ro, Seongbuk-gu, Seoul 02841, Republic of Korea

4 School of Mechanical Engineering, Korea University, Seoul 02841, Republic of Korea

\section{Introduction}

Gastric cancer remains the third most common cancer and a leading cause of mortality in the world. The highest incidence rates of gastric cancer occur in eastern Asia, Europe, and South America [1,2]. Despite improved treatment options, almost all patients with advanced gastric cancer eventually relapse [3]. However, molecular mechanisms of relapse and progression of gastric cancer remain largely unknown. Emerging studies have suggested that cancer stem cells (CSCs) contribute to cancer recurrence after treatment and are responsible for tumor evolution $[4,5]$.

Three-dimensional in vitro spheroid models are considered to be more physiologically relevant models of normal and diseased human tissues than are cells cultured in two dimensions [6]. Among numerous 3D models, spherical cancer models are characterized by morphology, presence of cancer cells, and capacity to be maintained 
as free-floating cultures [7]. Over the last several years, spheroid cultures that rely on the anchorage-independent growth properties of stem cells have been used to enrich CSCs in many solid tumors [8-12]. The spheroid formation method, particularly non-adherent conditions with serum-free media, has been used for most gastric cancer studies [10,13]. Cells in a spheroid directly interact with neighboring cells and secrete extracellular matrix into the environment [14]. These cells are also associated with maintenance of stemness, induction of epithelial-mesenchymal transition (EMT), and invasion [15, 16]. Spheroidforming cells are generally believed to conserve the characteristics, gene expression profiles, tumor heterogeneity, and tumor morphologies of their original tumors [12]. This implication suggests that spheroid formation can be considered a surrogate for human gastric cancer.

In 1965, Lauren described two distinct histologic classifications of gastric adenocarcinomas, intestinal type and diffuse type [17]. These two Lauren types of gastric adenocarcinoma have distinct clinical profiles [18]. The intestinal type of gastric cancer is more common in men and older patients, and is related to environmental factors such as Helicobacter pylori infection, diet, and lifestyle. In contrast, the diffuse type is more common in women and young patients, and is associated with genetic abnormalities [19]. Recently, The Cancer Genome Atlas (TCGA) network proposed a molecular classification dividing gastric cancer into the following four subtypes: Epstein-Barr virus (EBV)-positive tumors, microsatellite instable (MSI) tumors, genomically stable (GS) tumors, and tumors with chromosomal instability (CIN) [20]. The EBV and MSI subtypes of gastric cancer are associated with a vigorous immunological reaction and overexpression of immune checkpoint molecules. Therefore, these two subtypes of gastric cancer are particularly attractive candidates for immune checkpoint blockade. Trials in these particular gastric cancer subtypes are underway $[21,22]$. In contrast, the other two gastric cancer subtypes limit the effect of many therapeutic targets, including HER2 overexpression [23]. Other targeted therapeutics, including those directed against the signaling pathways of mitogenesis, angiogenesis, and immune checkpoints, are under clinical investigation for treatment of gastric cancer [23]. In addition, there are reports of gastric cancer subtypes with distinct gene expression patterns that respond to chemotherapy and are associated with patient survival [24]. Prior evaluation of the clinical and histological characteristics of these molecular subtypes suggests that the therapeutic strategy may depend on cancer subtype.

Considering the previous data, we hypothesized that spheroid-forming cells may have different characteristics classified by Lauren histological type. In this study, we isolated spheroid-forming cells from gastric cancer cell lines. We classified the spheroids according to histological type, assessed their gene expression patterns, and explored potential targeted treatments.

\section{Materials and methods}

\section{Cell lines and culture conditions}

The human gastric cancer cell lines AGS, SNU-216, SNU484, SNU-601, SNU-638, SNU-668, SNU-719, NCI-N87, MKN-1 and MKN-45 were obtained from the Korean Cell Line Bank (Seoul, Korea). The MKN-28 cell line was purchased from Riken (Ibaraki, Japan). Cells were maintained in RPMI-1640 medium (HyClone, Waltham, MA, USA) supplemented with 10\% fetal bovine serum (FBS) (HyClone), $25 \mathrm{mM}$ 4-(2-hydroxyethyl)-1-piperazineethanesulfonic acid (HEPES) (Invitrogen, Carlsbad, CA, USA), $25 \mathrm{mM} \mathrm{NaHCO}_{3}$ (Invitrogen), and 1\% penicillin/streptomycin (HyClone). Cells were grown at $37{ }^{\circ} \mathrm{C}$ in a humidified incubator with $5 \% \mathrm{CO}_{2}$ until the monolayers reached confluence.

\section{Spheroid forming assay}

Spheroid culture was performed as previously described [25]. Briefly, $5 \times 10^{3}$ parent cells were seeded onto a microwell plate (C-Wll, Incyto, Chonan, Korea), which was placed in a 60-mm Petri dish (SPL, Pocheon, Korea). The medium was changed every 4 days. After 2 weeks, the spheroids were examined using an Olympus CK40 optical microscope (Olympus, Tokyo, Japan). Diameter of spheroids was calculated using captured images. Spheroid cells larger than $75 \mu \mathrm{m}$ were considered as formed spheroids. For further studies, spheroids were dissociated into single cells using trypsin-EDTA and mechanical disruption with a pipette. The resulting cells were then suspended and counted for inoculation.

\section{Soft agar colony formation assay}

Soft agar assays were constructed in a $60-\mathrm{mm}$ dish as previously described [26]. Briefly, $5 \times 10^{3}$ cells were resuspended in $2 \mathrm{ml}$ with a final concentration of $1 \times$ media containing $0.3 \%$ agarose (Amresco, Solon, OH, USA). The cells were then spread onto a $2 \mathrm{ml}$ base layer containing $0.6 \%$ agar. The plates were incubated for $14-21$ days at $37{ }^{\circ} \mathrm{C}$ with $5 \%$ $\mathrm{CO}_{2}$. The plates were then stained with $0.01 \%$ crystal violet (Sigma) and 10\% methanol solution for $1 \mathrm{~h}$. The assays were repeated a total of three times. Colonies were calculated using bright field images and diameter larger than $100 \mu \mathrm{m}$ were counted. 


\section{Cell invasion assay}

For the invasion assay, the cells were loaded in the upper chamber of the Transwell culture inserts ( $8 \mu \mathrm{m}$ pore size) at a density of $2 \times 105$ cells/well. Subsequently, $200 \mu \mathrm{l}$ of serum-free RPMI medium was added to the upper chamber. A volume of $750 \mu \mathrm{l}$ of complete medium containing $10 \%$ FBS was added to the lower chamber. After incubation at $37{ }^{\circ} \mathrm{C}$ for $24 \mathrm{~h}$ in a $5 \% \mathrm{CO}_{2}$ incubator, noninvaded cells on the upper surface of the filter were removed with a cotton swab. Cells on the lower surface of the filter were fixed and stained with $4 \%$ paraformaldehyde and crystal violet. Three microscopic fields of view were randomly captured with a Canon EOS 600D CCD Camera (Canon). The assays were repeated a total of three times. The average number of cells was calculated and used for statistical analysis.

\section{Real-time quantitative PCR}

Total RNA was prepared using TRIzol (MRC, Cincinnati, $\mathrm{OH}$, USA) according to the manufacturer's instructions. For quantitative RNA analysis, $1 \mu \mathrm{g}$ of total RNA was reverse transcribed using M-MLV reverse transcriptase (Invitrogen) to produce cDNA. Amplification was performed in a CFX Real-Time PCR Detection System (Bio-Rad, Hercules, CA, USA) using iQ SYBR Green Supermix (Bio-Rad). For quantitative real-time PCR analysis, the expression level of each mRNA of interest was normalized to the GAPDH mRNA expression level. The following primer sequences were used:

OCT4: forward 5'-GCAGCGACTATGCACAACGA-3', reverse 5'-CCAGAGTGGTGACGGAGACA-3'; SOX2, forward 5'-CATCACCCACAGCAAATGACA-3', reverse 5'-GCTCCTACCGTACCACTAGAACTT-3'; NANOG, forward 5'-AATACCTCAGCCTCCAGCAGATG-3', reverse 5'-TGCGTCACACCATTGCTATTCTTC-3'; GAPDH, forward 5'-CAGCCTCAAGATCATCAGCAATG-3', reverse 5'-TCATGAGTCCTTCCACGATACCA-3'.

\section{Western blot analysis}

Western blot analysis was performed as previously described [25]. To collect tumor cell lysates, resected tumors were lysed in lysis buffer by bead beating using a Precellys 24 homogenizer (Bertin Technologies, Montigny-le-Bretonneux, France). The following concentrations were used for the primary antibodies: anti-OCT4, 1:1000 (Abcam, Cambridge, UK); anti-CD44, 1:1000 (Abcam); anti-SOX2, 1:500 (Cell Signaling Technology, Beverly, MA, USA); anti-NANOG, 1:1000 (Cell Signaling Technology); antiMMP9, 1:1000 (Cell Signaling Technology); anti-ERBB2, 1:1000 (Cell Signaling Technology); anti-ERBB3, 1:1000 (Cell Signaling Technology); anti-E-cadherin, 1:1000 (Santa Cruz Biotechnology); anti-c-Met, 1:1000 (Santa Cruz
Biotechnology); anti-EGFR. 1:1000 (Santa Cruz Biotechnology); anti-N-cadherin, 1:1000 (BD Bioscience, San Jose, CA, USA); anti-Snail, 1:1000 (EnoGene, Nanjing, Jiangsu, China); and anti-beta-actin, 1:10,000 (Sigma-Aldrich).

\section{Tet-inducible shRNA expression system}

Oligonucleotides were used to form tet-on inducible shRNA constructs with tet-on pLKO shRNA knockdown vectors (Addgene plasmid \#21915, Cambridge, MA, USA). TetpLKO-puro-Scrambled was a gift from Charles Rudin (Addgene plasmid \#47541). The shRNA hairpin targeting the 3'UTR was purchased from Macrogen (Seoul, Korea). The shRNA hairpin sequences are as follows: SOX2 (5'-CCGG-CTGCCGAGAATCCATGTATAT-CTCGAG -ATATACATGGATTCTCGGCAG-TTTTT-3'); E-cadherin (5'-CCGG-CCAGAGCTTCAAGACTGTTTA-CTCGAG -TAAACAGTCTTGAAGCTCTGG-TTTTT-3'); ERBB3 (5'-CCGG-GCAGAAATTATTGGGCTCTTT-CTCGAG -AAAGAGCCCAATAATTTCTGC-TTTTT-3'). The shRNA oligos were cloned into the AgeI and EcoRI sites in place of the stuffer fragment. All constructs were confirmed by DNA sequencing.

\section{Lentiviral cell transduction}

Lentiviral vector particles were produced by transfection of 293 T cells as previously described [27]. Two days after transfection, cell debris was removed using a $0.45-\mu \mathrm{m}$-poresized filter and subsequently concentrated through centrifugation using an Amicon ${ }^{\circledR}$ Ultra-15 Centrifugal filter unit (EMD Millipore, Billerica, MA, USA). The cells were infected with viral particles as previously described [28] and selected in puromycin.

\section{Xenograft model}

The xenograft studies were approved by the Institutional Animal Care and Use Committee (IACUC) of Korea University College of Medicine (IACUC approval No. KOREA2017-0108). Six-week-old male nude mice were purchased from Orient Bio Animal Center (Seongnam, Korea). The nude mice were injected with the indicated number of parent or spheroid cells subcutaneously into the flank. To establish tumor for Tet-on shRNA expression system, cells were subcutaneously injected $\left(1 \times 10^{7}\right.$ cells for MKN-28 and $4 \times 10^{6}$ cells for NCI-N87). Once the mean tumor volume reached $100 \mathrm{~mm}^{3}$, the mice were randomly divided into control and treatment groups. The groups were treated with a standard water supply or water supplemented with $1 \mathrm{mg} / \mathrm{mL}$ doxycycline and $2 \%$ sucrose for 21 days. 


\section{TCGA analysis}

TCGA datasets from 290 samples of stomach adenocarcinoma were analyzed for gene mutation and amplification using the GISTIC2 algorithm in the cbioportal platform (http://www.cbioportal.org). The platform was used to investigate the relationship between ERBB3 alteration and clinical outcome.

\section{Kaplan-Meier analysis}

The 5-year overall survival (OS) rates were analyzed for gastric cancer cases from a publicly available database (kmplot. com) [29]. A total of 320 patients were analyzed from the following datasets: GSE14210, GSE15459, GSE22377, GSE29272, GSE51105, and GSE62254. The input gene is ERBB3 (1563253_s_at). The analysis was restricted to Lauren classification intestinal $(n=320)$ and diffuse $(n=241)$ types of gastric cancers. Other subtypes included all patients (gender, perforation, treatment, and HER2 status).

\section{Statistical analysis}

Data are expressed as means \pm standard deviations. At least three experiments were performed independently for each type of experiment to ensure reproducibility. Statistical differences among the experimental groups were evaluated using analysis of variance, followed by Student's $t$ test. $P$ values $<0.05$ were considered statistically significant.

\section{Results}

\section{Isolation of spheroid-forming single cells enriched with stem-like properties}

Our group previously found that spheroid-forming single cells could be successfully isolated using a limited dilution protocol (with a microwell plate) [25]. We later focused on the characteristics of spheroid-forming cells with regard to colony formation, invasion, and tumorigenic ability. We found that spheroid-forming cells possessed a potent colony-forming ability on a soft agar assay (Fig. 1a). The spheroid-forming cells also had a greater invasive capacity than did parental cells using a transwell invasion assay (Fig. 1b). We used a MKN-28 gastric cancer cell line to determine whether spheroids have higher tumorigenic ability. The MKN-28 line was used because the SNU-638 cell line does not have tumorigenic ability (in either parent or spheroid cells) despite injection of at least one million cells (data not shown). In xenograft studies, we injected an equal number $\left(5 \times 10^{4}, 1 \times 10^{5}, 5 \times 10^{5}\right)$ of freshly dissociated spheroids and parental cells into subcutaneous flank tissue of the mice (four mice per group). After this injection, the spheroids generated larger size tumors than did the parent cells (Fig. 1c). Overall, these results provide evidence that isolated spheroid-forming cells exhibit stemlike properties in gastric cancer cell lines. a
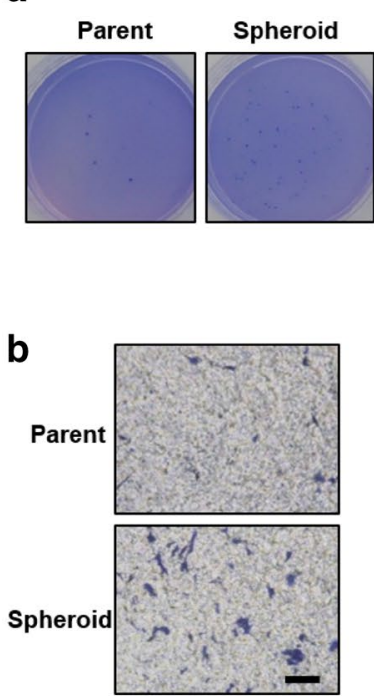
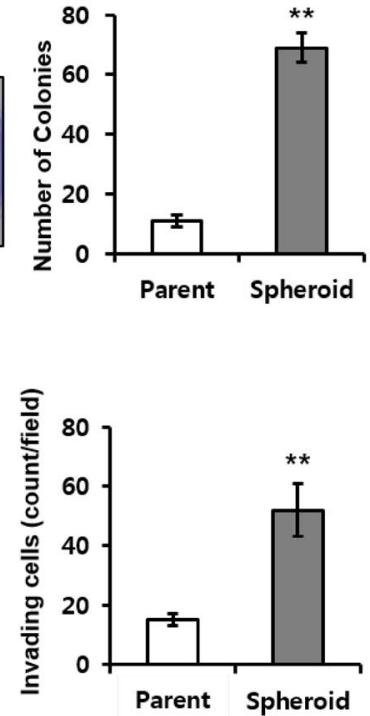

C

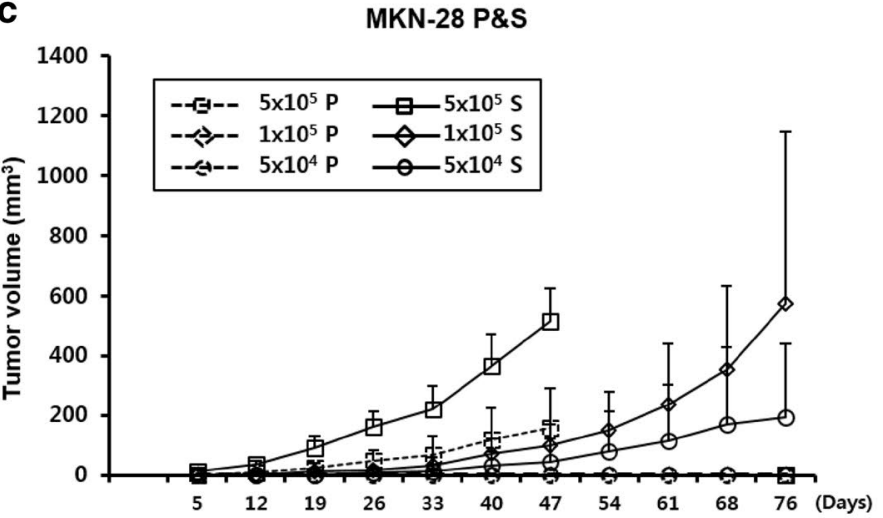

Fig. 1 Isolation of spheroid-forming cells and evaluation of the cancer stem-like phenotypes. a Soft agar colony-forming assay of SNU638 parent and spheroid cells, and statistical analysis. 2000 cells/dish. Day 20. b Transwell invasion assays of SNU-638 parent and spheroid cells. Cell invasion ability was calculated by counting cells per field.

c The growth curves showed that spheroids (S) resulted in a marked increase in tumor volume compared with the parent $(\mathrm{P})$ group. The error bars represent SD. Scale bar $=100 \mu \mathrm{m}$. ${ }^{* *} p<0.01$, compared to the parent group $(n=3)$ 


\section{Isolation of spheroid-forming cells from both types of gastric cancer cell lines}

Six intestinal-type and five diffuse-type gastric adenocarcinoma cell lines were selected to represent the Lauren classification. These lines were cultured to grow as spheroids, as described earlier. In the diffuse-type cell lines, SNU-484 and SNU-638 formed round spheroids with clear boundaries in several microwells (Fig. 2a). The spheroids were propagated to the same morphology when they were enzymatically and mechanically separated into single cells and seeded onto new microwell plates. However, all MKN-45 and SNU-601 cells formed loosely adherent cell clusters that could be easily dispersed by pipetting. These cell clusters were not considered appropriate spheroid-forming cells. In addition, the SNU-668 cells did not form spheroids. In the intestinal type, only the MKN-28 and NCI-N87 cell lines formed spheroids and could be propagated with further subculturing (Fig. 2a). In contrast, the MKN-1, SNU-216, SNU-719, and AGS lines did not form spheroids. Therefore, SNU-484, SNU-638, MKN-28, and NCI-N87 cell lines were chosen for further studies.

\section{Comparison of stemness gene expressions in both histological types of spheroid-forming cells}

Many studies have demonstrated that spheroid-forming cells were found to be enriched for cells with stem-like characteristics such as upregulation of three stem cell-specific transcription factors (OCT4, SOX2, and NANOG) [30,31].

a
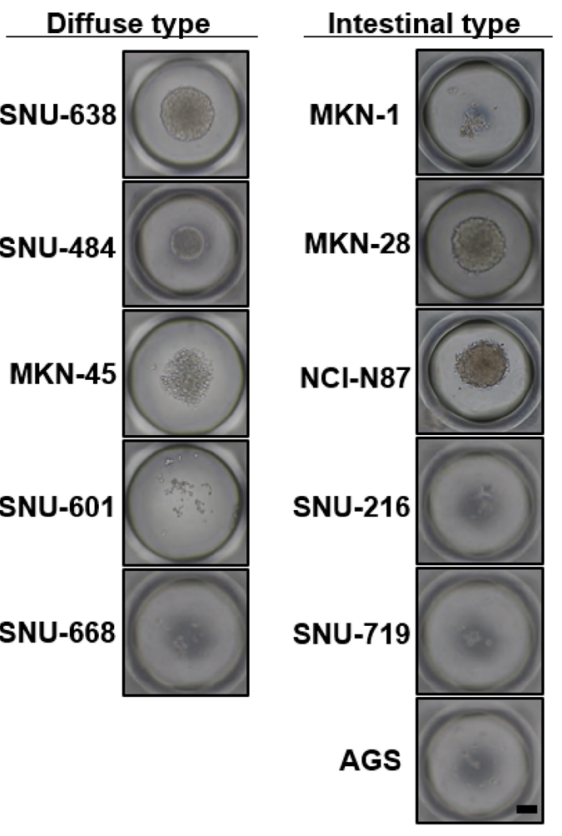

C

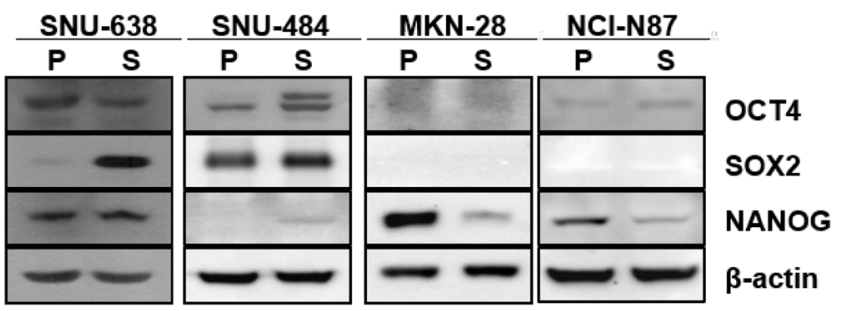

d

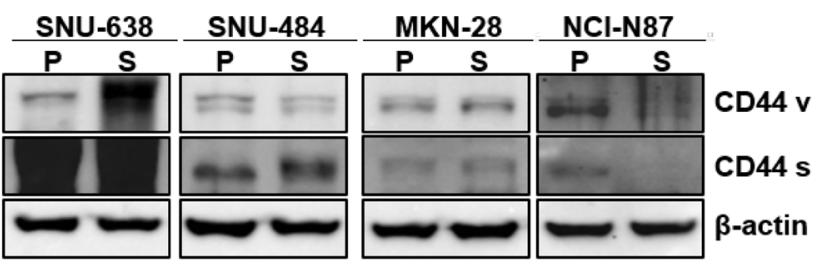

b
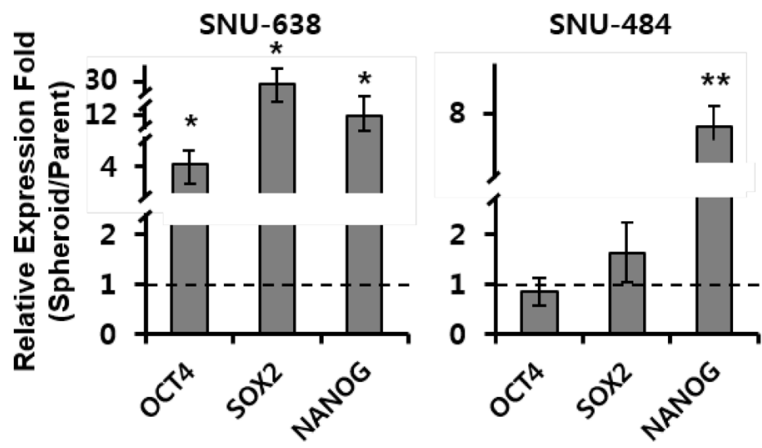

MKN-28

NCI-N87
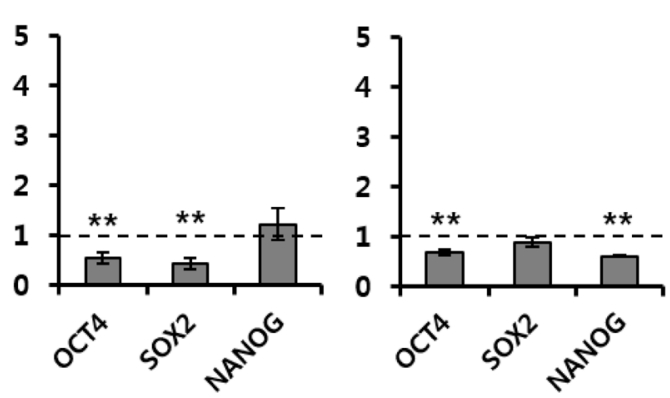

Fig. 2 Different characteristics of spheroid-forming cells in gastric cancer cell lines. a Isolation of single cell-derived spheroid cells of human gastric cancer cell lines included in the study. Microscopic images of spheroid culture after 2 weeks $(\times 40$ magnification, scale bar $=100 \mu \mathrm{m})$. b mRNA expression of stemness markers in sphe- roid cells as measured by qRT-PCR. Each data point represents the mean of three replicates. Error bars represent SD. Expression levels were normalized to that of GAPDH. c, d Western blot analysis of stemness proteins and CD44; beta-actin was used as a loading control. $* * p<0.01, * p<0.05$, compared to the parent group $(n=3)$ 
We examined whether the stemness gene expression differed across histological types of spheroid-forming cells. We first examined the levels of stemness gene expression in the two Lauren intestinal types and two Lauren diffuse types grown as monolayers or as spheroids. In the SNU-638 diffuse gastric cancer cell line, there were significantly increased mRNA levels of OCT4, SOX2, and NANOG in spheroid cells compared to those in parental cells (Fig. 2b). Consistent with these results, the protein levels of SOX2, but not OCT4 and NANOG were increased in spheroids (Fig. 2c). The transcription levels of SOX2 and NANOG in SNU-484 spheroids were higher than parental cells (Fig. 2b). And those protein expression levels in SNU-484 spheroids were increased compared to the parental cells (Fig. 2c). However, these levels in the intestinal type (MKN-28 and NCI-N87) were comparable between spheroid cells and parental cells. The expression of SOX2 increased when cells were grown to spheroids, particularly in the spheroid-forming cells of diffuse-type cell lines (SNU-638 and SNU-484).

CD44 expression has also been described to produce stem-like properties [10,32]. Following separation of spheroid-forming cells, we found that CD44 expression increased in the diffuse type but not in intestinal type of cancer (Fig. 2d). The CD44 variant isoform expression was significantly elevated in SNU-638 spheroids, whereas CD44 standard isoform expression was elevated in SNU-484 spheroids. These results suggest the existence of a distinct malignant pathway for the diffuse and intestinal types of spheroid-forming cells in gastric cancer. Among the genes investigated, we selected SOX2 as an outstanding candidate gene that can potentially maintain self-renewal capability in diffuse type spheroid-forming cells.

\section{Identification of differential gene expressions between intestinal and diffuse types of spheroid-forming cells}

According to the TCGA subtype of gastric cancer, GS tumors, which are commonly seen in diffuse histology, exhibited elevated expression of cell adhesion molecule. In addition, CIN tumors, which are commonly found on intestinal histology, develop their key features as a result of genomic amplification of receptor tyrosine kinases (RTKs). Therefore, we decided to examine the changes in expression of major RTK proteins and EMT-related proteins, which are correlated with cell adhesion and cell-cell junction.

We first examined the EMT- and RTK-related protein expression in parental and spheroid cells from four gastric cancer cell lines. Using immunoblot analysis, we found that the SNU-638 and SNU-484 diffuse-type spheroid cells had higher expression of E-cadherin than did parental cells (Fig. 3a). MKN-28 and NCI-N87, two intestinal
Fig. 3 The major protein expression related with EMT and RTKs in parental and spheroid cells from four gastric cancer cell lines. a Western blot analysis of EMT-related proteins. b Western blot analysis of RTK-related proteins. Beta-actin was used as a loading control a
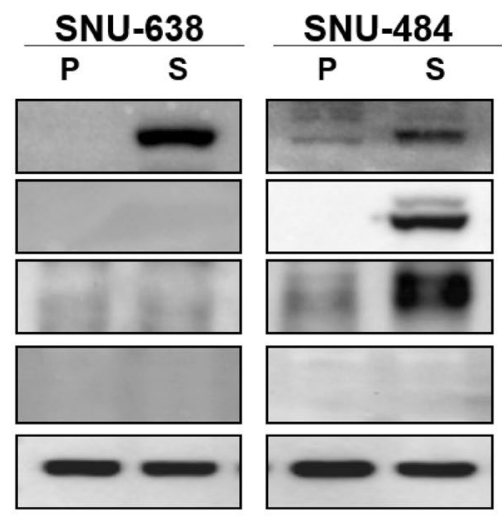

b

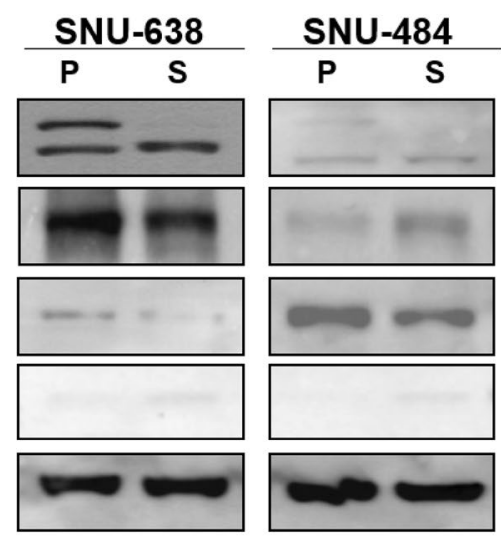

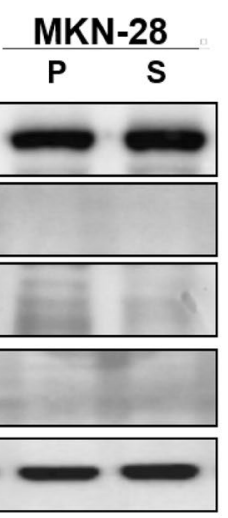

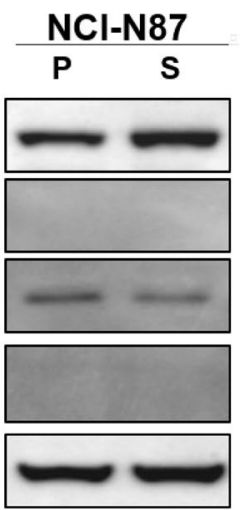

E-cadherin

$\mathrm{N}$-cadherin

SNAIL

MMP9

$\beta$-actin

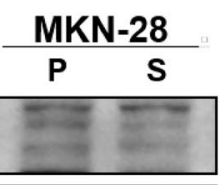

NCl-N87

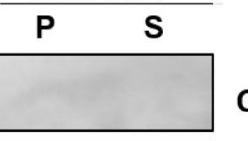

C-Met
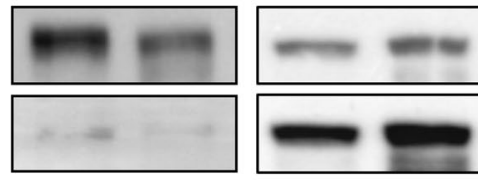

EGFR
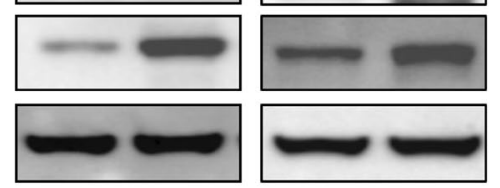

HER2

ERBB3

$\beta$-actin 
type spheroid cell lines, exhibited a higher expression of ERBB3 than did parental cells (Fig. 3b). In contrast, there were no differences in MET and EGFR expression between parental cells and spheroid cells. However, NCIN87, in which the ERBB2 gene is highly amplified and its protein product is expressed at very high levels as previously reported [33]. These data suggest that E-cadherin plays an important role in the maintenance of diffuse-type spheroid-forming cells. Similarly, ERBB3 might play a critical role in intestinal-type spheroid-forming cells.

\section{Growth inhibition of spheroid cells in diffuse type by downregulation of SOX2 and E-cadherin expression}

To investigate the effects of SOX2 and E-cadherin on spheroid cell growth and anchorage-independent growth, we suppressed target gene expression using a Tet-inducible shRNA hairpin construct. Inhibition of SOX2 and E-cadherin proteins was observed on doxycycline-induced shRNA knockdown (Fig. 4a). Depletion of SOX2 and E-cadherin in SNU-484 and SNU-638 diffuse-type cells potently inhibited spheroid growth, resulting in decreased spheroid size and numbers (Fig. 4b-d). As previously described, the SNU-638 cell line a

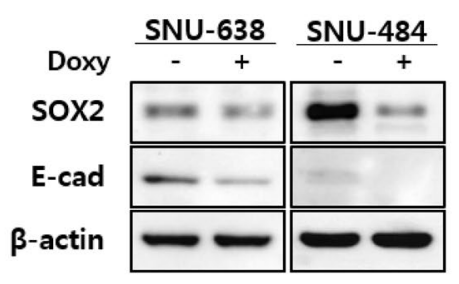

C

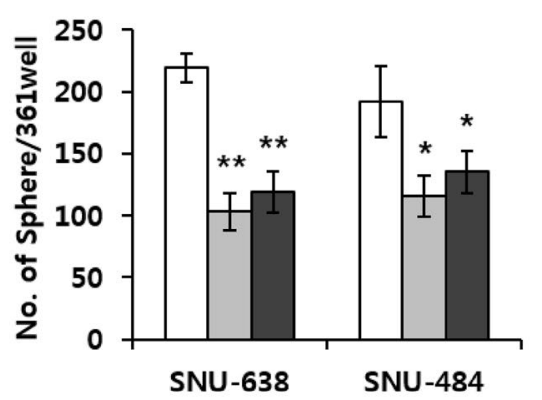

e

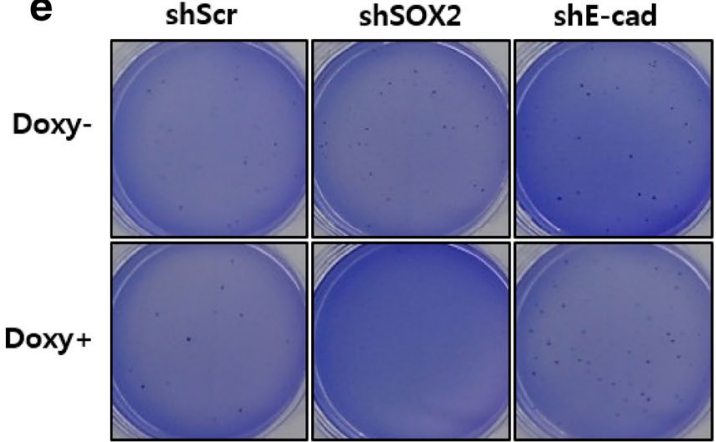

Fig. 4 Knockdown of SOX2 and E-cadherin decreased the growth of diffuse-type spheroid-forming cells. a Immunoblot showing reduction in SOX2 and E-cadherin protein after doxycycline (doxy) treatment (72 h, $500 \mathrm{ng} / \mathrm{mL}$ ) of SNU-638 and SNU-484 cells stably transduced with inducible shRNA. b Representative images of spheroid-forming cells induced with doxycycline in microwell plates and allowed to form spheroids for 14 days. Nontargeting scrambled (shScr) was b

SNU-638
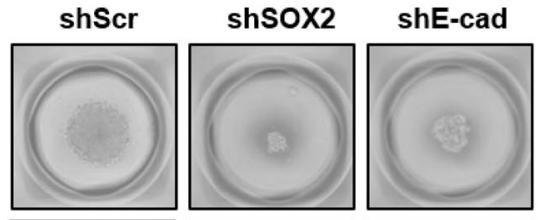

SNU-484
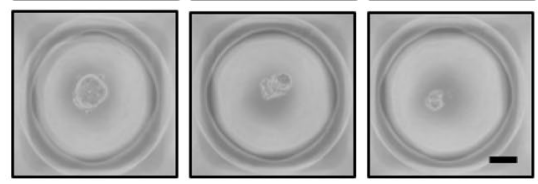

d

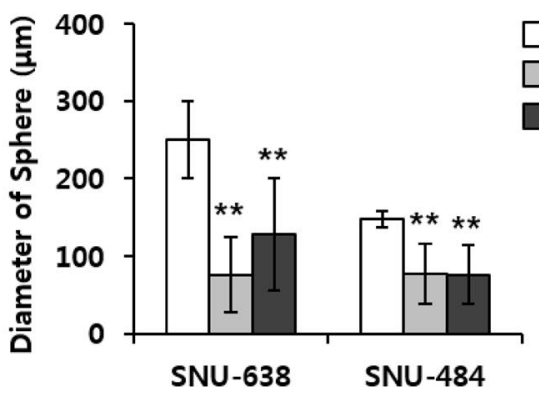

included $(\times 40$ magnification, scale bar $=100 \mu \mathrm{m})$. c Number of spheroids was quantified with diameters larger than $75 \mu \mathrm{m}$. d Spheroid diameter was measured on a spheroid image. e Soft agar assay of SNU-638 cells with or without doxycycline treatment. The graph represents the mean number of colonies $\pm \mathrm{SD}$. from experiments performed in triplicates. ${ }^{*} p<0.01,{ }^{*} p<0.05$, compared to the parent group $(n=3)$ 
did not show tumorigenic ability. We further investigated SOX2 and E-cadherin silencing on anchorage-independent growth by assessing colony formation on soft agar. Soft agar is the gold standard assay for cellular transformation in vitro [34]. Subsequently, we observed that doxycycline-induced SOX2 knockdown significantly reduced the number of colonies on soft agar compared to that of the controls (Fig. 4e). Conversely, E-cadherin depletion on the soft agar assay led to increased number of colonies in the SNU-638 cell line. The SNU-484 cell line did not allow colony formation in the soft agar assay, even after 40 days of incubation in both parent and spheroid cells. These results indicate that SOX2, but not E-cadherin, plays an important role in anchorage-independent growth in diffuse-type spheroid-forming cells.

\section{Reducing ERBB3 expression decreased tumorigenicity of intestinal-type spheroid cells}

We generated a cell line with tet-inducible shRNA to explore the function of ERBB3 in intestinal-type spheroid-forming cells (Fig. 5a). As expected, spheroid growth in MKN-28 and NCI-N87 cells was significantly inhibited by inducible knockdown of ERBB3, resulting in decreased spheroid size and numbers (Fig. 5b-d). We also evaluated the effect of depleting ERBB3 in intestinal-type spheroid cells. Similarly, ERBB3 knockdown resulted in significant inhibition of colony formation in the intestinal-type spheroid cells (Fig. 5e).

To determine whether ERBB3 knockdown could suppress tumor growth, we inoculated mice with MKN-28 and NCI-N87 cells expressing inducible ERBB3 shRNA. When the xenograft tumors reached a volume of $100 \mathrm{~mm}^{3}$, the mice were randomly divided into two groups and treated with doxycycline or standard water supply for 21 days. The doxycycline-treated tumors grew more slowly than the untreated tumors. After 3 weeks, the doxycycline-treated tumors harbored decreased tumor cellularity compared to that of the untreated tumors (Fig. 6a, b, d). Immunohistochemistry confirmed decreased levels of ERBB3 and Ki67, and lower cell survival in resected tumors treated with doxycycline compared to those in untreated tumors (Fig. 6d). In addition, the tumors isolated from the treated group had not only reduced ERBB3 expression, but also lower phosphor Akt expression than the untreated group. These results suggest that ERBB3 plays a role in tumor growth through the Akt pathway (Fig. 6c). These data revealed that targeting ERBB3 has an in vivo antitumor effect in intestinal-type spheroid-forming cells.

\section{Validation of ERBB3 gene alterations and clinical significance in a public dataset}

ERBB3 expression is critical for tumor growth (in the xenograft model) in intestinal-type spheroid-forming cells.
Therefore, we further analyzed its effect on clinical outcomes of 290 gastric cancer tissues via cBioPortal [35]. We observed that intestinal-type gastric adenocarcinoma exhibited a high frequency of genetic alterations of ERBB3 $(23.8 \%, 46 / 193)$ (Fig. 7a). However, in diffuse-type gastric adenocarcinoma, those gene alterations were uncommon, with a rate of $5.9 \%, 4 / 67$. Furthermore, ERBB3 amplification was only observed in intestinal-type gastric adenocarcinoma. In addition, missense ERBB3 mutations (unknown, putative driver) had high scores in functional analysis through the hidden Markov model prediction and were considered 'pathogenic' [36] (data not shown). The ERBB3 mRNA expression was significantly greater in the intestinal type than in the diffuse-type group (Fig. 7b).

We next analyzed the association between gene alteration and patient outcome. The Kaplan-Meier survival analysis demonstrated that high level of ERBB3 was strongly associated with poor prognosis in gastric cancer (Fig. 7c) in the intestinal type, but not in the diffuse type (data not shown). High ERBB3 expression was associated with poor survival in gastric adenocarcinoma, suggesting that it plays an important role in intestinal-type tumor development and can predict clinical outcomes.

\section{Discussion}

Although important advances have been made in the molecular classification of gastric cancer, there are still limited therapeutic options for this malignancy. Because of the different clinical and histological characteristics of gastric cancer subtypes, it is important to identify new therapeutic targets for future clinical approaches in cancer treatment.

The aim of this study was to investigate whether spheroid-forming cells possess different characteristics based on subtype. For this purpose, we employed spheroid-forming methods that have been widely used as powerful tools for isolating self-renewal cells. However, the conventional spheroid bodies themselves display marked cellular heterogeneity; therefore, experiments using spheroids should be interpreted as studies of mixed cell populations [37]. Therefore, we used a microwell-based culture chip, which is the most suitable approach for isolating more homogeneous spheroidforming cells from a single cell [25].

This study demonstrated that spheroid-forming gastric cancer cells showed differential gene expressions depending on histological type. These results correspond with the findings of earlier studies, which showed that SOX2 is a stem cell-related gene in many solid cancers and correlates with the clinicopathologic factors in gastric cancer [38-40]. However, our results are also different from other previous studies. We observed increased expression level of SOX2 in the diffuse type of spheroid-forming cells, but not in 
a

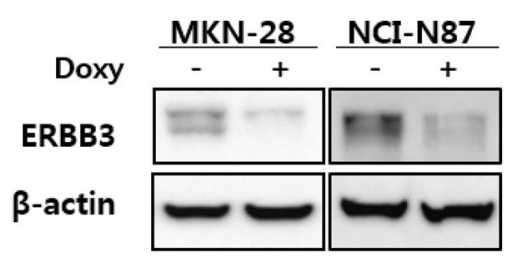

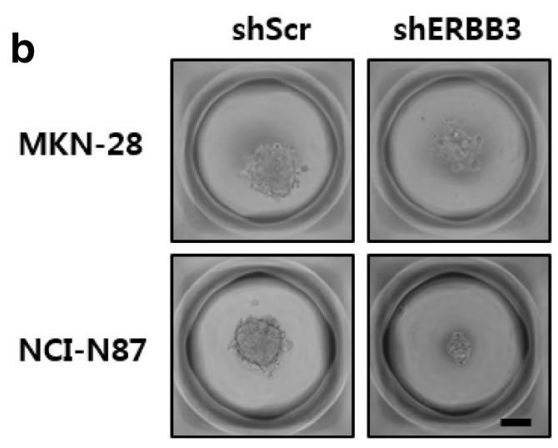

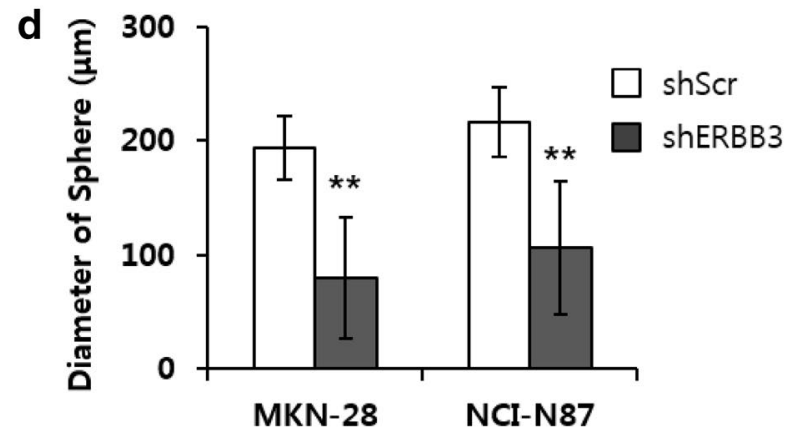

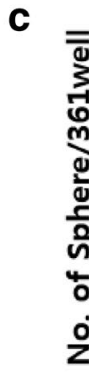

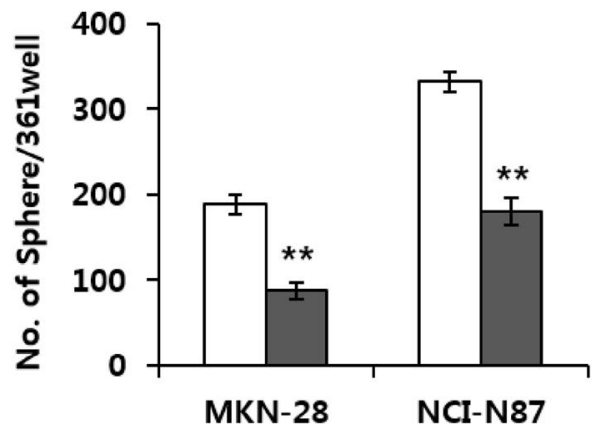

e

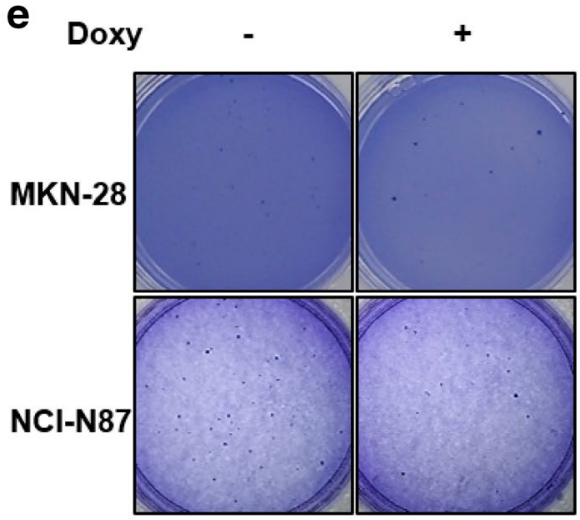

Fig. 5 ERBB3 knockdown diminished the growth of diffuse-type spheroid-forming cells. a Immunoblot showing reduction of ERBB3 protein after doxycycline (doxy) treatment $(72 \mathrm{~h}, 500 \mathrm{ng} / \mathrm{mL})$ in MKN-28 and NCI-N87 cells stably transduced with inducible shRNA. b Representative images of spheroid-forming cells induced with doxycycline in microwell plates and allowed to form spheroids for 14 days $(\times 40$ magnification, scale bar $=100 \mu \mathrm{m})$. Nontarget-

the intestinal type. Likewise, CD44, which is the most frequently reported cell surface marker for gastric CSCs, only demonstrates an increased expression in the diffuse type. These results support our hypothesis that spheroid cells may have different characteristics according to histological type. Therefore, we additionally investigated differently expressed genes linked to histological type.

E-cadherin is one of the cell surface molecules that functions with calcium-dependent adhesion [41]. Low E-cadherin expression in gastric cancer tissue suggests that it plays ing scrambled (shScr) was included. $\mathbf{c}$ The number of spheroids was quantified using diameters larger than $75 \mu \mathrm{m}$. d Spheroid diameter was measured on a spheroid image. e Soft agar assay of MKN-28 and NCI-N87 cells with or without doxycycline treatment. Graph shows the mean number of colonies \pm SD from experiments performed in triplicates. ${ }^{*} p<0.01,{ }^{*} p<0.05$, compared to the parent group $(n=3)$

a critical role in cancer development and progression [42]. In addition, $\mathrm{CDH} 1$ mutations are considered to be common somatic alterations in diffuse gastric cancer [43]. In our analysis, increased expression of E-cadherin appeared to correlate with the diffuse type of spheroid cells, but now with the intestinal type. There are similar reports that spheroid-forming ability depends upon E-cadherin expression [44]. Therefore, we presume that increased expression of E-cadherin might be related to the ability to grow as spheroids in SNU-638 and SNU-484 cells. 
a

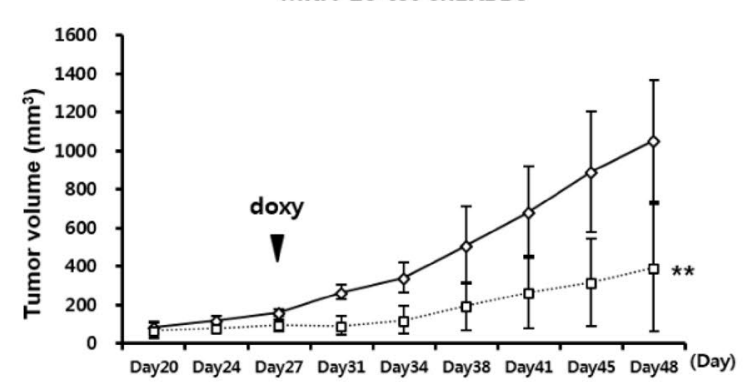

NCI-N87 tet-shERBB3

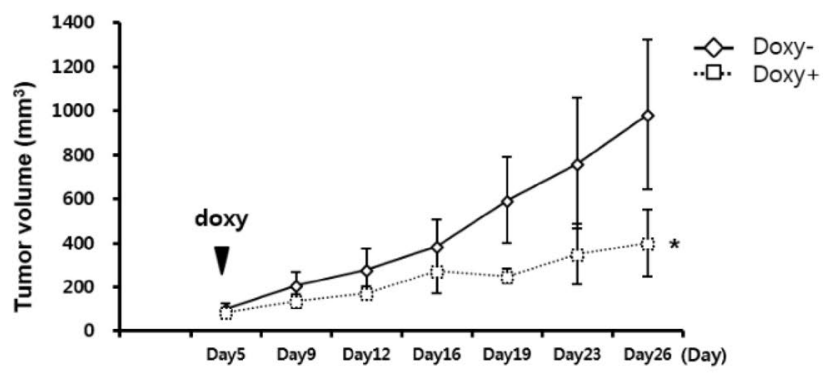

MKN-28 tet-shERBB3 NCI-N87 tet-shERBB3

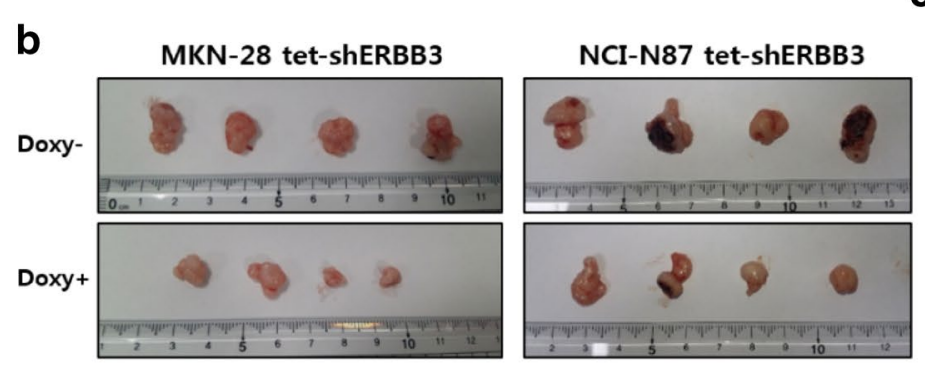

C Doxy $\frac{-}{\# 1 \quad \# 2} \frac{+}{\# 1 \quad \# 2}$

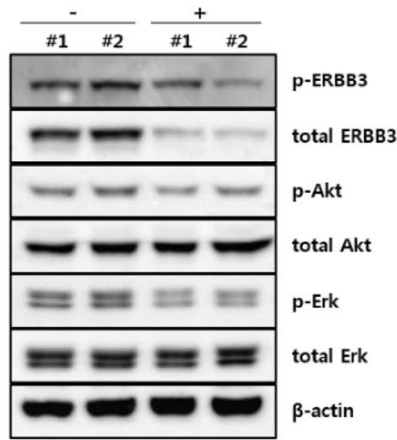

d

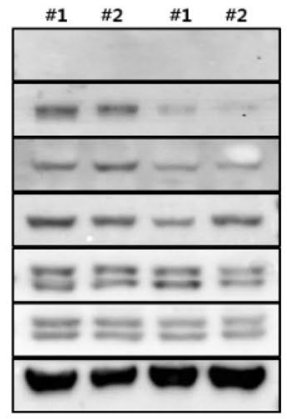

MKN-28 tet-ShERBB3

Doxy+

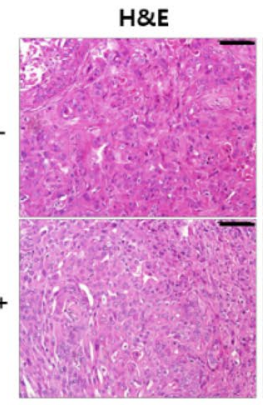

ERBB3
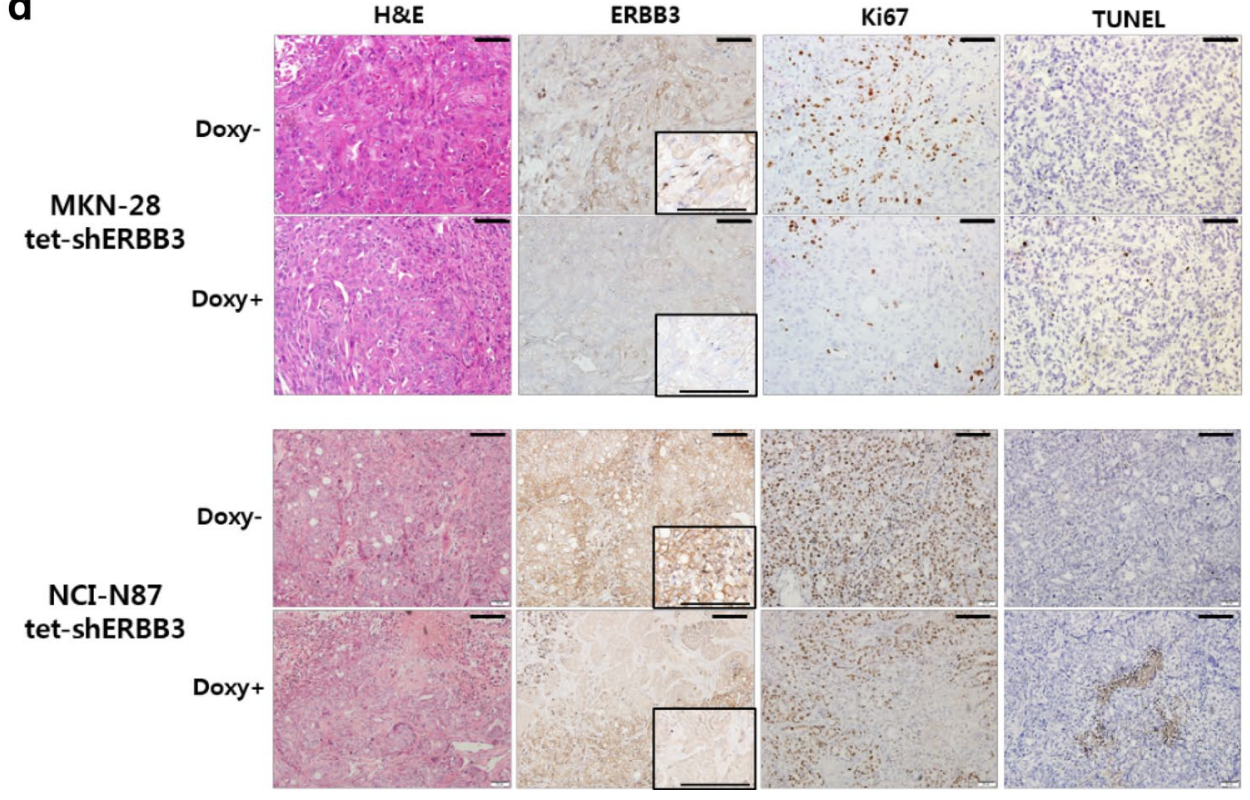

Fig. 6 Inducible Tet-mediated ERBB3 ablation decreases tumor growth. a Balb/c nude male mice were treated with doxycycline for 21 days $(1 \mathrm{mg} / \mathrm{mL}$ in drink water) until the tumor surpassed $100 \mathrm{~mm}^{3}$. Tumor volume was measured twice weekly. Values shown represent the average SD. $p$ values were calculated using Student's $t$ test, $N=4$. $* * p<0.01, * p<0.05$, compared to the parent group $(n=4)$. b Image of tet-shERBB3 tumor samples resected from mice in each group. c Lysates made from two tumors isolated from con-

trol and doxycycline-treated tumor-bearing-independent mice were subjected to immunoblotting. d H\&E analysis of tumors treated with doxycycline for 21 days demonstrate decreased tumor cellularity. ERBB3, Ki67, and cell survival were all lower in doxycycline-treated samples compared to those of untreated samples. TUNEL analysis revealed increased cell death in doxycycline-treated tumors compared to that of untreated samples $(\times 20$ magnification, scale bar $=100 \mu \mathrm{m})$

ERBB3 plays an important role in various cancer proliferation and survival of various cancer types [45]. ERBB3 does not possess a functional kinase activity at its cytoplasmic

domain. Therefore, it depends on dimerization with other ERBB members to produce a downstream signal [46]. ERBB3 overexpression is strongly associated with tumor 


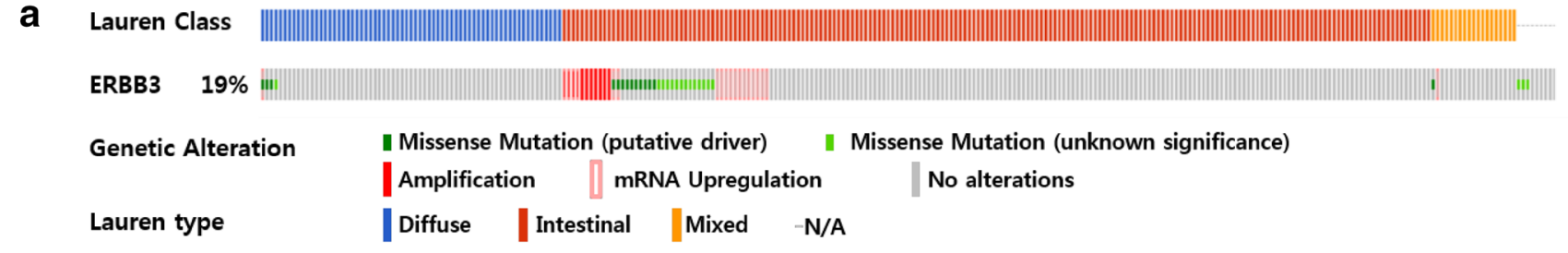

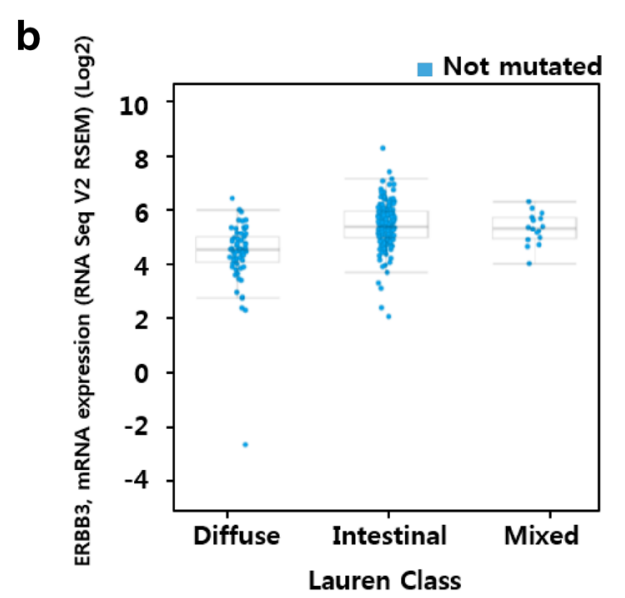

Fig. 7 ERBB3 has worse prognosis value in intestinal-type gastric cancer. a OncoPrint of ERBB3 genetic alteration in 290 gastric cancer samples based on TCGA data. b Box plot showing the association between mRNA levels and gene amplification. The horizontal line within the box indicates the median. Boundaries of the box indicate the 25th and 75th percentiles. The whiskers indicate the highest and

progression and poor prognosis in patients with gastric cancer [47]. Moreover, expression of ERBB3 was more frequently observed in the intestinal type than it was in the diffuse type [48]. The clinical relevance of the gastric cancer subtypes with distinct gene expression patterns is supported by the finding of increased ERBB3 expression in the intestinal type and in spheroid-forming cells. In this regard, it will be interesting to compare the classification to Lauren's histological subtypes. In our analysis, patients stratified by Lauren's histological subtype from a public data set had significantly different survival outcomes. Our data suggest that classification using spheroid-forming method might not only provide prognostic or predictive value, but also suggest therapeutic targets.

To demonstrate whether ERBB3 downregulation can suppress tumor growth, we attempted to directly inhibit ERBB3 in spheroid-forming MKN-28 cells. Knockdown of target genes in the spheroid cells using lentiviral particles containing pLKO anti-ERBB3 shRNA and shCont did not generate tumors when inoculated subcutaneously into nude mice. To explain this result, we hypothesized that the decreased tumorigenic ability in spheroid-forming MKN-28 cells may be toxic to the xenograft model. Therefore, we applied stable

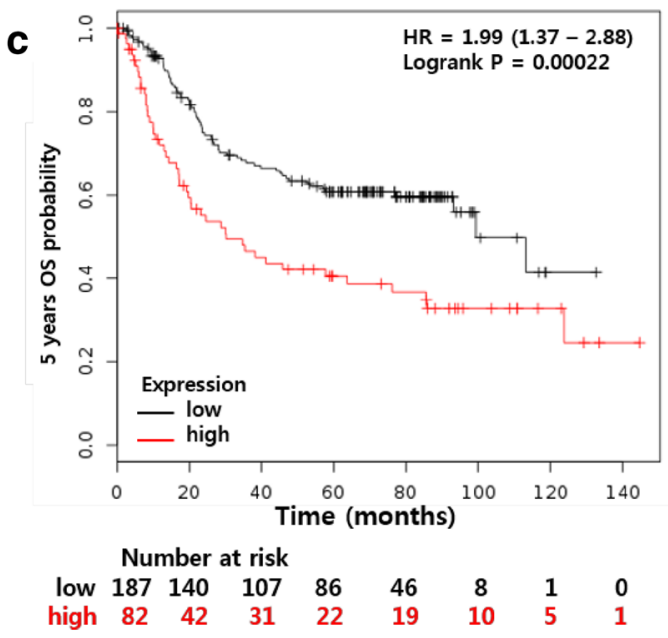

lowest values of the results. c Five-year overall survival graphs of intestinal-type gastric cancer based on ERBB3 expression. Using a publicly available database (KM plotter; http://www.kmplot.com), a total of 320 patients were intestinal type; probe $=1563253$ _s_at; Logrank $p$ values were calculated in kmplot database

cell and conditional shRNA expression systems to knockdown ERBB3 in the MKN-28 and NCI-N87 xenograft tumor model. For this reason, the xenograft model is limited in its evaluation of the direct efficacy of ERBB3 downregulation in spheroid cells. However, we also found that ERBB3 downregulation (using inducible ERBB3 shRNA) led to slower tumor growth in the treated group compared to that of the control group. These findings imply that downregulation of ERBB3 plays a role in inhibition of tumor growth in the intestinal-type MKN-28 and NCI-N87 cell lines. Ultimately, these findings were obtained using isolated cell lines. Future studies are needed to substantiate these findings using spheroid-forming cells from primary tissue.

\section{Conclusion}

Our results suggest that the candidate target gene expression pattern of spheroid-forming cells is closely related to the cancer histological type. These data contribute to understanding of the characteristics of each subtype of spheroidforming cells in gastric cancer and suggest target genes for its treatment. 
Acknowledgements This study was supported by a Grant from the National Research Foundation of Korea (NRF), which is funded by the Korean government (MEST) (No. 2010-0020986).

\section{Compliance with ethical standards}

Conflict of interest The authors declare that they have no conflicts of interest.

Animal studies All institutional and national guidelines for the care and use of laboratory animals were followed.

\section{References}

1. Torre LA, Bray F, Siegel RL, Ferlay J, Lortet-Tieulent J, Jemal A. Global cancer statistics, 2012. CA Cancer J Clin. 2015;65:87-108.

2. Jung KW, Won YJ, Kong HJ, Lee ES. Cancer statistics in Korea: incidence, mortality, survival, and prevalence in 2015. Cancer Res Treat. 2018;50:303-16.

3. Hohenberger P, Gretschel S. Gastric cancer. Lancet. 2003;362:305-15.

4. Visvader JE, Lindeman GJ. Cancer stem cells: current status and evolving complexities. Cell Stem Cell. 2012;10:717-28.

5. Magee JA, Piskounova E, Morrison SJ. Cancer stem cells: impact, heterogeneity, and uncertainty. Cancer Cell. 2012;21:283-96.

6. Ivanov DP, Grabowska AM. Spheroid arrays for high-throughput single-cell analysis of spatial patterns and biomarker expression in 3D. Sci Rep. 2017;7:41160.

7. Weiswald LB, Bellet D, Dangles-Marie V. Spherical cancer models in tumor biology. Neoplasia. 2015;17:1-15.

8. Fang D, Nguyen TK, Leishear K, Finko R, Kulp AN, Hotz S, et al. A tumorigenic subpopulation with stem cell properties in melanomas. Cancer Res. 2005;65:9328-37.

9. Wright MH, Calcagno AM, Salcido CD, Carlson MD, Ambudkar SV, Varticovski L. Brca1 breast tumors contain distinct CD44+/ CD24- and CD133+ cells with cancer stem cell characteristics. Breast Cancer Res. 2008;10:R10.

10. Takaishi S, Okumura T, Tu S, Wang SS, Shibata W, Vigneshwaran $\mathrm{R}$, et al. Identification of gastric cancer stem cells using the cell surface marker CD44. Stem Cells. 2009;27:1006-20.

11. Todaro M, Alea MP, Di Stefano AB, Cammareri P, Vermeulen L, Iovino $\mathrm{F}$, et al. Colon cancer stem cells dictate tumor growth and resist cell death by production of interleukin-4. Cell Stem Cell. 2007;1:389-402.

12. Qureshi-Baig K, Ullmann P, Rodriguez F, Frasquilho S, Nazarov $\mathrm{PV}$, Haan S, et al. What do we learn from spheroid culture systems? Insights from tumorspheres derived from primary colon cancer tissue. PLoS One. 2016;11:e0146052.

13. Song Z, Yue W, Wei B, Wang N, Li T, Guan L, et al. Sonic hedgehog pathway is essential for maintenance of cancer stem-like cells in human gastric cancer. PLoS One. 2011;6:e17687.

14. Fennema E, Rivron N, Rouwkema J, van Blitterswijk C, de Boer J. Spheroid culture as a tool for creating 3D complex tissues. Trends Biotechnol. 2013;31:108-15.

15. Han XY, Wei B, Fang JF, Zhang S, Zhang FC, Zhang HB, et al. Epithelial-mesenchymal transition associates with maintenance of stemness in spheroid-derived stem-like colon cancer cells. PLoS One. 2013;8:e73341.

16. Davidowitz RA, Selfors LM, Iwanicki MP, Elias KM, Karst A, Piao $\mathrm{H}$, et al. Mesenchymal gene program-expressing ovarian cancer spheroids exhibit enhanced mesothelial clearance. J Clin Investig. 2014;124:2611-25.
17. Lauren P. The two histological main types of gastric. Carcinoma: diffuse and so-called intestinal-type carcinoma. An attempt at a histo-clinical classification. Acta Pathol Microbiol Scand. 1965;64:31-49.

18. Lynch HT, Grady W, Suriano G, Huntsman D. Gastric cancer: new genetic developments. J Surg Oncol. 2005;90:114-33 (discussion 33)

19. Hu B, El Hajj N, Sittler S, Lammert N, Barnes R, Meloni-Ehrig A. Gastric cancer: classification, histology and application of molecular pathology. J Gastrointest Oncol. 2012;3:251-61.

20. TCGA. Comprehensive molecular characterization of gastric adenocarcinoma. Nature. 2014;513:202-9.

21. Wang M, Busuttil RA, Pattison S, Neeson PJ, Boussioutas A. Immunological battlefield in gastric cancer and role of immunotherapies. World J Gastroenterol. 2016;22:6373-84.

22. Kim ST, Cristescu R, Bass AJ, Kim KM, Odegaard JI, Kim $\mathrm{K}$, et al. Comprehensive molecular characterization of clinical responses to PD-1 inhibition in metastatic gastric cancer. Nat Med. 2018;24:1449-58.

23. Kankeu Fonkoua L, Yee NS. Molecular characterization of gastric carcinoma: therapeutic implications for biomarkers and targets. Biomedicines. 2018;6:32.

24. Tan IB, Ivanova T, Lim KH, Ong CW, Deng N, Lee J, et al. Intrinsic subtypes of gastric cancer, based on gene expression pattern, predict survival and respond differently to chemotherapy. Gastroenterology. 2011;141:476-85 (85.e1-11).

25. Lee JW, Sung JS, Park YS, Chung S, Kim YH. Isolation of spheroid-forming single cells from gastric cancer cell lines: enrichment of cancer stem-like cells. Biotechniques. 2018;65:197-203.

26. Zhi QM, Chen XH, Ji J, Zhang JN, Li JF, Cai Q, et al. Salinomycin can effectively kill ALDH(high) stem-like cells on gastric cancer. Biomed Pharmacother. 2011;65:509-15.

27. Lee JS, Hmama Z, Mui A, Reiner NE. Stable gene silencing in human monocytic cell lines using lentiviral-delivered small interference RNA. Silencing of the p110alpha isoform of phosphoinositide 3-kinase reveals differential regulation of adherence induced by 1 alpha,25-dihydroxycholecalciferol and bacterial lipopolysaccharide. J Biol Chem. 2004;279:9379-88.

28. Losman JA, Looper RE, Koivunen P, Lee S, Schneider RK, McMahon C, et al. (R)-2-hydroxyglutarate is sufficient to promote leukemogenesis and its effects are reversible. Science. 2013;339:1621-5.

29. Lanczky A, Nagy A, Bottai G, Munkacsy G, Szabo A, Santarpia L, et al. miRpower: a web-tool to validate survival-associated miRNAs utilizing expression data from 2178 breast cancer patients. Breast Cancer Res Treat. 2016;160:439-46.

30. Liu J, Ma L, Xu J, Liu C, Zhang J, Liu J, et al. Spheroid bodyforming cells in the human gastric cancer cell line MKN-45 possess cancer stem cell properties. Int J Oncol. 2013;42:453-9.

31. Wang AM, Huang TT, Hsu KW, Huang KH, Fang WL, Yang MH, et al. Yin Yang 1 is a target of microRNA-34 family and contributes to gastric carcinogenesis. Oncotarget. 2014;5:5002-16.

32. Lau WM, Teng E, Chong HS, Lopez KA, Tay AY, Salto-Tellez $\mathrm{M}$, et al. CD44v8-10 is a cancer-specific marker for gastric cancer stem cells. Cancer Res. 2014;74:2630-41.

33. Chang HR, Park HS, Ahn YZ, Nam S, Jung HR, Park S, et al. Improving gastric cancer preclinical studies using diverse in vitro and in vivo model systems. BMC Cancer. 2016;9:200.

34. Rotem A, Janzer A, Izar B, Ji Z, Doench JG, Garraway LA, et al. Alternative to the soft-agar assay that permits high-throughput drug and genetic screens for cellular transformation. Proc Natl Acad Sci USA. 2015;112:5708-13.

35. Gao J, Aksoy BA, Dogrusoz U, Dresdner G, Gross B, Sumer SO, et al. Integrative analysis of complex cancer genomics and clinical profiles using the cBioPortal. Sci Signal. 2013;6:p11. 
36. Shihab HA, Rogers MF, Gough J, Mort M, Cooper DN, Day IN, et al. An integrative approach to predicting the functional effects of non-coding and coding sequence variation. Bioinformatics. 2015;31:1536-43.

37. Basu S, Campbell HM, Dittel BN, Ray A. Purification of specific cell population by fluorescence activated cell sorting (FACS). J Vis Exp. 2010. https://doi.org/10.3791/1546.

38. Leis O, Eguiara A, Lopez-Arribillaga E, Alberdi MJ, Hernandez-Garcia S, Elorriaga K, et al. Sox 2 expression in breast tumours and activation in breast cancer stem cells. Oncogene. 2012;31:1354-65.

39. Boumahdi S, Driessens G, Lapouge G, Rorive S, Nassar D, Le Mercier M, et al. SOX2 controls tumour initiation and cancer stem-cell functions in squamous-cell carcinoma. Nature. 2014;511:246-50.

40. Hutz K, Mejias-Luque R, Farsakova K, Ogris M, Krebs S, Anton M, et al. The stem cell factor SOX2 regulates the tumorigenic potential in human gastric cancer cells. Carcinogenesis. 2014;35:942-50.

41. van Roy F, Berx G. The cell-cell adhesion molecule E-cadherin. Cell Mol Life Sci. 2008;65:3756-88.

42. Zhang J, Chen XY, Huang KJ, Wu WD, Jiang T, Cao J, et al. Expression of FoxM1 and the EMT-associated protein E-cadherin in gastric cancer and its clinical significance. Oncol Lett. 2016;12:2445-50.

43. Becker KF, Hofler H. Frequent somatic allelic inactivation of the E-cadherin gene in gastric carcinomas. J Natl Cancer Inst. 1995;87:1082-4.
44. Manuel Iglesias J, Beloqui I, Garcia-Garcia F, Leis O, VazquezMartin A, Eguiara A, et al. Mammosphere formation in breast carcinoma cell lines depends upon expression of E-cadherin. PLoS One. 2013;8:e77281.

45. Mujoo K, Choi BK, Huang Z, Zhang N, An Z. Regulation of ERBB3/HER3 signaling in cancer. Oncotarget. 2014;5:10222-36.

46. Pinkas-Kramarski R, Soussan L, Waterman H, Levkowitz G, Alroy I, Klapper L, et al. Diversification of Neu differentiation factor and epidermal growth factor signaling by combinatorial receptor interactions. EMBO J. 1996;15:2452-67.

47. Hayashi M, Inokuchi M, Takagi Y, Yamada H, Kojima K, Kumagai J, et al. High expression of HER3 is associated with a decreased survival in gastric cancer. Clin Cancer Res. 2008;14:7843-9.

48. He XX, Ding L, Lin Y, Shu M, Wen JM, Xue L. Protein expression of HER2, 3, 4 in gastric cancer: correlation with clinical features and survival. J Clin Pathol. 2015;68:374-80.

Publisher's Note Springer Nature remains neutral with regard to jurisdictional claims in published maps and institutional affiliations. 